VoL. 51 (1995) [369-375]

\title{
EXTENSION OF HÖLDER'S INEQUALITY (I)
}

\author{
E.G. KwoN
}

A continuous form of Hölder's inequality is established and used to extend the inequality of Chuan on the arithmetic-geometric mean inequality.

1.

Throughout we let $X=(X, \mathcal{S}, \mu)$ and $Y=(Y, \mathcal{T}, \nu)$ be $\sigma$-finite measure spaces with positive measures $\mu$ and $\nu$. When we call $f$ defined on $X \times Y$ measurable it refers to $(\mathcal{S} \times \mathcal{T})$-measurable. $\mu \times \nu$ denotes the product measure of $\mu$ and $\nu$ (see [4, Chapter 7]). $L^{1}(\mu)$ denotes the space of Lebesgue integrable (with respect to $\mu$ ) functions defined on $\mathrm{X}$ and $L^{p}(\mu), 0<p<\infty$, denotes the space of those complex measurable $f$ defined on $X$ for which $|f|^{p} \in L^{1}(\mu)$.

In Section 2 we establish a continuous form of Hölder's inequality. In Section 3 we give an application of the inequality by generalising a result of Chuan [2] on the arithmetic-geometric mean inequality. In Section 4, we give further extensions of the result of Section 3.

\section{2.}

If $0 \leqslant x \leqslant 1$, then Hölder's inequality says that

$$
\int_{Y} f_{1}(y)^{x} f_{2}(y)^{1-x} d \nu(y) \leqslant\left(\int_{Y} f_{1}(y) d \nu(y)\right)^{x}\left(\int_{Y} f_{2}(y) d \nu(y)\right)^{1-x}
$$

for all positive functions $f_{1}$ and $f_{2}$ of $L^{1}(\nu)$. It is known that (2.1) can be extended to the case of a multiple product of functions (see, for example, [1, 3 and 5$]$ ), and even to a countable product of functions (provided the product converges). In this section, we generalise (2.1) to the following

Theorem 1. (Continuous form of Hölder's inequality). Let $\mu(X)=1$. Let $f(x, y)$ be a positive measurable function defined on $X \times Y$. Then

$$
\int_{Y} \exp \left(\int_{X} \log f d \mu\right) d \nu \leqslant \exp \left\{\int_{X} \log \left(\int_{Y} f d \nu\right) d \mu\right\}
$$

Received 31st May, 1994

This work was supported by the Korea Ministry of Education under BSRI-94-1411, and by KOSEF.

Copyright Clearance Centre, Inc. Serial-fee code: 0004-9729/95 SA2.00+0.00. 
Equality holds in (2.2) as a nonzero finite value if and only if

$$
f(x, y)=g(x) h(y) \quad \text { almost everywhere } \mu \times \nu
$$

for a positive $\mu$-measurable function $g$ with $-\infty<\int_{X} \log g d \mu<\infty$ and a positive $\nu$-measurable $h$ with $\int_{Y} h d \nu=1$.

Proof: To avoid the difficulty caused by measure zero sets and $\sigma$-finiteness of $Y$, we shall use the well known fact that if $\mu(X)=1$ and $F$ is a positive function in $L^{1}(\mu)$ then

$$
\lim _{p \rightarrow 0}\left(\int_{X} F^{p} d \mu\right)^{1 / p}=\exp \left(\int_{X} \log F d \mu\right)
$$

and the convergence is monotone $[4$, p.74].

If $\nu(Y)=0$ there is nothing to prove. Otherwise, let $\left\{Y_{n}\right\}$ be a measurable sequence of subsets of $Y$ such that

$$
Y_{1} \subset Y_{2} \subset \ldots, \bigcup_{n=1}^{\infty} Y_{n}=Y, \nu\left(Y_{n}\right)<\infty
$$

and let

$$
f_{N}(x, y)= \begin{cases}\min \{f(x, y), N\} & \text { if } y \in Y_{N} \\ 0 & \text { otherwise } .\end{cases}
$$

If (2.2) is valid for $f_{N}$ and $Y_{N}$ instead of $f$ and $Y$ then (2.2) follows from the monotone convergence theorem by taking the limit as $N \rightarrow \infty$. Therefore we may assume that $0<\nu(Y)<\infty$ and $f \in L^{1}(\mu \times \nu)$. Now, setting $F(x)=f(x, y)$ in (2.3), we have

$$
\lim _{p \rightarrow 0}\left(\int_{X} f(x, y)^{p} d \mu(x)\right)^{1 / p}=\exp \left(\int_{X} \log f(x, y) d \mu(x)\right)
$$

almost everywhere $d \nu$. On integrating both sides of (2.4) with respect to $d \nu$, we obtain

$$
\int_{Y} \exp \left(\int_{X} \log f(x, y) d \mu(x)\right) d \nu(y)=\int_{Y} \lim _{p \rightarrow 0}\left(\int_{X} f(x, y)^{p} d \mu(x)\right)^{1 / p} d \nu(y) .
$$

Since $\left(\int_{X} f(x, y)^{p} d \mu(x)\right)^{1 / p}$ is decreasing as $p \rightarrow 0$, by the monotone convergence theorem this last term equals

$$
\lim _{p \rightarrow 0} \int_{Y}\left(\int_{X} f(x, y)^{p} d \mu(x)\right)^{1 / p} d \nu(y) .
$$


On the other hand, if we use the continuous form of Minkowski's inequality, we have

$$
\int_{Y}\left(\int_{X} f(x, y)^{p} d \mu(x)\right)^{1 / p} d \nu(y) \leqslant\left\{\int_{X}\left(\int_{Y} f(x, y) d \nu(y)\right)^{p} d \mu(x)\right\}^{1 / p}
$$

provided $0<p \leqslant 1$. Taking the limit $p \rightarrow 0$ on both sides of (2.6) and using (2.3) once more, we bound (2.5) by

$$
\exp \left\{\int_{X} \log \left(\int_{Y} f(x, y) d \nu(y)\right) d \mu(x)\right\} .
$$

This proves (2.2).

If

$$
f(x, y)=g(x) h(y) \quad \text { almost everywhere } \mu \times \nu
$$

for some positive measurable functions $g$ and $h$ with $-\infty<\int_{X} \log g d \mu<\infty$ and $\int_{Y} h d \nu=1$ then it is not difficult to see that equality holds in (2.2) as a nonzero finite value. Conversely, suppose that equality holds in (2.2) as a nonzero finite value. Then

$$
\int_{Y} \exp \left(\int_{X} \log \frac{f}{\int_{Y} f d \nu} d \mu\right) d \nu=1 \text {. }
$$

Since Jensen's inequality and Fubini's inequality tell us that

$$
\int_{Y} \exp \left(\int_{X} \log \frac{f}{\int_{Y} f d \nu} d \mu\right) d \nu \leqslant \int_{Y}\left(\int_{X} \frac{f}{\int_{Y} f d \nu} d \mu\right) d \nu=1,
$$

to have (2.7) we should have equality in (2.8):

$$
\int_{Y}\left\{\exp \left(\int_{X} \log \frac{f}{\int_{Y} f d \nu} d \mu\right)-\left(\int_{X} \frac{f}{\int_{Y} f d \nu} d \mu\right)\right\} d \nu=0 .
$$

Hence the inner integral of (2.9) is zero almost everywhere $[\nu]$, so that

$$
\frac{f}{\int_{Y} f d \nu}=\text { constant function of } x:=h(y)
$$

almost everywhere $x \in X$ once $y \in Y$ is fixed [ $\nu$ ]-almost everywhere. If we set $\int_{Y} f d \nu=$ $g(x)$ then $g$ is positive $\mu$-measurable (see [4, Theorem 7.8]) and $-\infty<\int_{X} \log g d \mu<$ $\infty$. Also, $h$ is positive $\nu$-measurable. Therefore

$$
f(x, y)=g(x) h(y) \quad \text { almost everhwhere } \mu \times \nu
$$

with $g, h$ positive measurable, $\int_{Y} h d \nu=1$ and $-\infty<\int_{X} \log g d \mu<\infty$. This completes the proof.

If we take

$$
X=\{1,2\}, f(x, y)=f_{x}(y), x \in X,
$$

and

$$
d \mu=\left(t \chi_{\{1\}}+(1-t) \chi_{\{2\}}\right) d m,
$$

$d m$ the counting measure, $\chi_{\{.\}}$the corresponding characteristic functions, then (2.2) reduces to (2.1). 
3.

Concerning the arithmetic-geometric mean inequality, Chuan [2] inserted a continuum of additional terms between the two sides of the inequality as follows.

Theorem A. If $n$ is a natural number, $s>0, a_{j}>0, q_{j}>0(j=1, \ldots, n)$, and $q_{1}+\ldots+q_{n}=1$ then

$$
\prod_{j=1}^{n} a_{j}^{q_{j}} \leqslant\left(s \int_{0}^{\infty}\left[\prod_{j=1}^{n}\left(x+a_{j}\right)^{q_{j}}\right]^{-s-1} d x\right)^{-1 / s} \leqslant \sum_{j=1}^{n} q_{j} a_{j}
$$

Our result in this section is the following generalisation of Theorem A:

Theorem 2. If $s>0, \mu(X)=1$, and $f$ is a positive function of $L^{1}(\mu)$, then

$$
G_{X} f \leqslant\left\{s \int_{0}^{\infty} \exp \left(\int_{X} \log (y+f(x))^{--1} d \mu(x)\right) d y\right\}^{-1 / s} \leqslant A_{X} f
$$

Either of the equalities in (3.2) holds if and only if $f(x)$ is constant almost everywhere $[\mu]$.

Here

$$
G_{X} f=\exp \left(\int_{X} \log f(x) d \mu(x)\right)
$$

and

$$
A_{X} f=\int_{X} f(x) d \mu(x)
$$

are respectively the geometric mean and the arithmetic mean of $f$ over $X$.

Proof: Besides using Theorem 1, our proof is modelled from that of Theorem A in [2]. By Jensen's inequality

$$
\exp \left(\int_{X} \log (y+f(x)) d \mu(x)\right) \leqslant \int_{X}(y+f(x)) d \mu(x)=y+A_{X} f
$$

for all $y>0$. Thus for $s>0$

$$
\begin{aligned}
\int_{0}^{\infty}\left\{\exp \left(\int_{X} \log (y+f(x)) d \mu(x)\right)\right\}^{-\infty-1} d y & \geqslant \int_{0}^{\infty}\left(y+A_{X} f\right)^{--1} d y \\
& =\frac{1}{s}\left(A_{X} f\right)^{-\bullet}
\end{aligned}
$$

(3.3) proves the second inequality of (3.2). 
To prove the first inequality of (3.2), apply Theorem 1 to get

$$
\begin{aligned}
\int_{0}^{\infty}\left\{\exp \left(\int_{X} \log (y+f(x)) d \mu(x)\right)\right\}^{-s-1} d y \\
\quad \leqslant \exp \left\{\int_{X} \log \left(\int_{0}^{\infty}(y+f(x))^{-s-1} d y\right) d \mu(x)\right\} \\
\quad=\exp \left(\int_{X} \log \frac{1}{s} f(x)^{-s} d \mu(x)\right) \\
\quad=\frac{1}{s} \exp \left(\int_{X} \log f(x) d \mu(x)\right)^{-s}=\frac{1}{s}\left(G_{X} f\right)^{-s}
\end{aligned}
$$

which is the desired result.

Next, in order to have equality in either one of the inequalities of (3.2), we should have equality either in (3.3) or in (3.4). By considering the equality case of Jensen's inequality and that of (2.2) as stated in Theorem 1, we conclude that $f$ is constant almost everywhere $[\mu]$. The proof is complete.

4.

Let's denote, for positive $\mu$-measurable function $f$,

$$
M_{p} f=M_{p}(f, X)=\left(\int_{X} f^{p}(x) d \mu(x)\right)^{1 / p}
$$

if $0<p<\infty$ and

$$
M_{0} f=\exp \left(\int_{X} \log f(x) d \mu(x)\right) .
$$

Note that $M_{1}(f)=A_{X} f$ and $M_{0}(f)=G_{X} f$ provided $\mu(X)=1$. Our goal in this section is to improve (3.2) as follows:

TheOREM 3. Suppose $s>0, \mu(X)=1$, and $f$ is positive $\mu$-measurable. If $0<p \leqslant q \leqslant 1$ and if $f \in L^{1}(\mu)$ then

$$
\begin{aligned}
M_{0} f & \leqslant\left\{s \int_{0}^{\infty} \exp \left(\int_{X} \log (y+f(x))^{-\bullet-1} d \mu(x)\right) d y\right\}^{-1 / \bullet} \\
& \leqslant 2^{(1 / p-1)(1 / \bullet+1)} M_{p} f
\end{aligned}
$$

and

$$
M_{p} f \leqslant\left\{s \int_{0}^{\infty}\left(\int_{X}(y+f(x))^{p} d \mu(x)\right)^{(-s-1) / p} d y\right\}^{-1 / \rho} \leqslant 2^{(-1+1 / q)(1+1 / x)} M_{q} f
$$


If $1 \leqslant q \leqslant p<\infty$ and if $f \in L^{p}(\mu)$ then we have (4.2) with both inequalities reversed.

Proof: Suppose that $0<p \leqslant q \leqslant 1$. The left side inequality of (4.1) follows from (3.2), and the right side inequality of (4.1) follows from (4.2) via the inequality

$$
\begin{aligned}
\left\{s \int_{0}^{\infty} \exp \left(\int_{X} \log (y+f(x))^{-s-1} d \mu(x)\right) d y\right\}^{-1 / \bullet} & \\
& \leqslant\left\{s \int_{0}^{\infty}\left(\int_{X}(y+f(x))^{p} d \mu(x)\right)^{(-\bullet-1) / p} d y\right\}^{-1 / s} .
\end{aligned}
$$

The last inequality is obvious by the convexity of exp $x$.

Now we prove (4.2). It follows from Minkowski's inequality that

$$
\left(\int_{X}(y+f(x))^{p} d \mu(x)\right)^{1 / p} \geqslant y+M_{p} f
$$

Hence

$$
s \int_{0}^{\infty}\left(\int_{X}(y+f(x))^{p} d \mu(x)\right)^{(--1) / p} d y \leqslant s \int_{0}^{\infty}\left(y+M_{p} f\right)^{--1} d y=\left(M_{p} f\right)^{-1}
$$

Therefore

$$
\left\{s \int_{0}^{\infty}\left(\int_{X}(y+f(x))^{p} d \mu(x)\right)^{(-s-1) / p} d y\right\}^{-1 / s} \geqslant M_{p} f
$$

On the other hand, since $0<p \leqslant q \leqslant 1$ and $\mu(X)=1$, we have

$$
\begin{aligned}
\left\{\int_{X}(y+f(x))^{p} d \mu(x)\right\}^{1 / p} & \leqslant\left\{\int_{X}(y+f(x))^{q} d \mu(x)\right\}^{1 / q} \\
& \leqslant\left\{\int_{X}\left(y^{q}+f^{q}(x)\right) d \mu(x)\right\}^{1 / q} \\
& \leqslant 2^{-1+1 / q}\left(y+M_{q} f\right) .
\end{aligned}
$$

Hence it follows that

$$
\begin{aligned}
\left\{s \int_{0}^{\infty}\left(\int_{X}(y+f(x))^{p} d \mu(x)\right)^{(--1) / p} d y\right\}^{-1 / \alpha} & \\
& \leqslant\left\{s \int_{0}^{\infty}\left(2^{-1+1 / q}\left(y+M_{q} f\right)\right)^{-/-1} d y\right\}^{-1 / 8} \\
& =2^{(-1+1 / q)(1+1 / 0)} M_{q} f
\end{aligned}
$$

Now (4.2) follows from (4.4) and (4.6). The proof of the other half, that is, of the case $1 \leqslant q \leqslant p<\infty$, proceeds exactly the same way by reversing the inequalities in (4.3), (4.4), (4.5) and (4.6). 


\section{REFERENCES}

[1] E.F. Beckenbach and R. Bellman, Inequalities (Springer-Verlag, Berlin, Heidelberg, New York, 1961).

[2] Hao Zhi Chuan, 'Note on the inequality of the arithmetic and geometric means', Pacific J. Math. 143 (1990), 43-46.

[3] G.H. Hardy, J.E. Littlewood and G. Pólya, Inequalities (Cambridge University Press, Cambridge, 1952).

[4] W. Rudin, Real and complex analysis (McGraw-Hill, New York, 1974).

[5] A.W. Roberts and D.E. Varberg, Convex functions (Academic Press, New York, 1973).

Department of Mathematics Education

Andong National University

Andong 760-749

Korea 